\title{
A Peer Review of the Assessment of Knowledge and Treatment Seeking Behavior among Tuberculosis and Multi-Drug Resistant Tuberculosis Patients: A Case Control Study
}

\author{
Article by Francis Farai Chikuse ${ }^{1}$, Pathcare Namibia ${ }^{2}$ \\ ${ }^{1}$ PHD Public Health Student of Texila America University \\ E-mail: francis.chikuse@gmail.com ${ }^{1}$
}

\begin{abstract}
Source: Yadav, S.K. et al. (2015). Assessment of Knowledge and Treatment Seeking Behavior among Tuberculosis (TB) and Multi-Drug Resistant Tuberculosis (MDR-TB) Patients. A Case Control Study. IOSR Journal of Dental and Medical Sciences (IOSR-JDMS). Volume 14, Issue 7 Ver. II, PP 66-71. Viewed on 10 August 2018: www.iosrjournals.org
\end{abstract}

\begin{abstract}
This review is an intellectual plot line of an article by Dr. Shiv Kumar Yadav et al., (2015) titled "Assessment of Knowledge and Treatment Seeking Behavior among Tuberculosis (TB) and MultiDrug Resistant Tuberculosis (MDR-TB) Patients". The researchers used unmatched case control study to explore the knowledge on causes, mode of spread, duration of treatment among drug susceptible TB (controls) and MDR-TB (cases) participants. A pre-tested, standardized semistructured questionnaire was used to collect data which was presented in tables and graphs. In this review, attempt will be made to provide a constructive, supportive and fair assessment of the article based on how it is written. This study revealed that there is wide gap in knowledge and treatment initiation among drug susceptible TB and drug resistant TB patients. Furthermore, this review will analyze the structure, content and relevance of the article in addressing knowledge and health seeking behavior among MDR-TB and TB patients. Numerous articles (Finnie et al., 2011, Skordis-Worrall, Hanson \& Mills, 2010; Mavhu et al, 2010; Yimer, Bjune \& Alene, 2005) have focused on diagnosis and treatment initiation delays among TB patients. Therefore, this review will identify both the strengths and weaknesses of this article with respect to this subject area and will proffer some supportive suggestions for improvement.
\end{abstract}

keywords: Drug Sensitive Testing, Health Seeking Behavior, Unmatched Case Control Study, Knowledge, Multi-Drug Resistant Tuberculosis, Drug Susceptible Tuberculosis, Treatment.

\section{Article structure and summary}

The article provides an abstract subdivided into context of the subject matter; setting and design; methods and materials; results and conclusion. Using purposive sampling technique, 68 cases were selected from the indoor ward of the Department of Pulmonary Medicine and 136 controls were drawn from patients registered for direct observed TB treatment in Surat District (rural and urban). The study was conducted between October 2013 and June 2014. Drug sensitivity testing was carried out at DTC Surat. A molecular technique in the form of cartridge based nucleic acid amplification test (CBNAAT) method was used to detect rifampicin resistance and susceptibility. Patient with rifampicin resistance TB were selected as cases and those with rifampicin sensitive TB were enrolled as controls.

Participation in the study was voluntary and those willing to participate had their sputum samples tested for drug resistance and susceptibility without coercion. For the respondents who were less than 18 years of age, participation was granted only if reliable parent (mother or father or guardian) was accompanying the respondent. The parents also gave consent for the respondents. In a separate study on TB health seeking behavior, Skordis-Worrall, Hanson and Mills (2010) excluded children with TB from participating in the study. Historically, children with TB have not been given much attention by researchers, policy makers and clinicians as the disease was thought to be less prevalent in children. 
This exclusion of children with TB and MDR-TB in most cases results in failure of interven tions to control epidemic TB. Children provide a significant pool of reservoir of TB and MDR-TB bacilli. Therefore, it is crucial that research efforts must also be redirected to identify, diagnose and treat TB in children. Being a case study, Yadav and colleagues state one of the study limitations as recall, interview and selection bias. Consequently, the findings of this study cannot be generalized. The article has links to references, citation, subjects and author allowing the reader to easily review and evaluate the article.

The authors used Strengthening the Reporting of Observation Studies in Epidemiology (STROBE) checklist. STROBE relates to a collaborative initiative of epidemiologists, researchers, statisticians, journal editors involved in carrying out research and reporting of observational studies at an international level. The authors presented the following: study setting, duration, collection tool, sample technique, sample size factoring in confounding factors. Additionally, an outline of the selection of cases and controls and their inclusion and exclusion criteria, assumption and ethical consideration are presented. However, the article fell short of describing how missing data was addressed, the source of funding and the role of funders in the study. There is need to identify cases of $\mathrm{TB}$ and MDR-TB within the communities using active case finding approach. The study used a passive (health center based) case finding model.

The article also failed to evaluate the relationship between patient, health system delay factors and MDR-TB using credible parametric or non-parametric tests. A statistical test like two-sided univariate analysis would have been significant to establish relationship among factors and demographics. No demographic data is given in this article. There questions probed from participants about their knowledge of TB were not exhaustive. The authors could have gathered information on modes of spread of TB (e.g. speaking, sharing of spoons, sneezing and coughing) and the participants' knowledge on TB symptoms e.g. cough, cough up blood, weight loss, fever and night and difficulty in breathing.

\section{Literature review}

Geographically, 22 high TB burden countries are developing countries where $25 \%$ of the poorest in world population lives and World Health Organization (WHO: 2013) states that this account for 80\% of the global TB cases. The setting of this article is Surat District in India. WHO (2013) avers that India and China have the largest number of TB cases estimated at $26 \%$ and $12 \%$ respectively of the world's total TB cases. In this region, understanding a host of factors that are closely associated with TB diagnosis and treatment delays is crucial in TB control interventions. Numerous studies on TB patients' diagnosis and treatments delays have found a number of factors (Finnie et al, 2011). Patient delay factors include stigma associated with human immunodeficiency virus (HIV) test (Macho et al., 2010), self-belief that TB symptoms like cough can either resolve or improve with self-medication (Mavhu et al, 2010) and visits to multiple health care providers (Skordis-Worrall, Hanson \& Mills, 2010). These studies, although not specific to MDR-TB, have assisted in identifying factors which contributes to patient delays in seeking health care.

Health provider delays include turnaround time of TB results, accuracy and sensitivity of the diagnostic testing methods, patient management between different health care providers and quality of specimens send to laboratories for investigations (Cowan, 2013). Most studies evaluated by Finnie et $a l$, (2011) used a cross-sectional design. Cross-sectional studies have a number of inherent limitations that potentially bias the results, such as low comparability and inclusion of certain confounders. Consequently, there is a challenge to obtain the causality of the observed associations among various variables. Yadav and colleagues used a method superior to cross-sectional design in form of a case control study, this method allows comparability and can establish causality among affected factors. However, another limitation of the article is that there is no distinction between patients based and provider/health system-based delays. The article could have assessed both patient and health system delays as components of total delay. Total delay resembles the patient and the health system delays from the time of first symptoms to the start of treatment. 


\section{Article critique}

\section{Author}

Yadav and colleagues are stated as resident doctors and professors in the Department of Community Medicine, GMC Surat, India. This improves their credibility as researchers. They communicated ideas using direct and straight forward words and phrases. The writing of the article is in such a way that there are no meandering commas and it's easy to find the verbs and adjectives that remain open and easy to understand in sentences. Rather the authors successfully communicated ideas and this is hall mark of academic research today.

\section{Accuracy}

The authors presented a convincing line of argument assessing the level of knowledge and treatment seeking behavior among MDR-TB and TB patients. They used current and credible references including data from India's National TB Control Programme 2010 report. This source contextualizes the status of drug susceptible and drug resistant TB in India. The authors assert that prevalence rate of TB in India is $40 \%$ and deaths almost occur in every 3 minutes. Thus, prevention and control of tuberculosis need a number of interventions addressing patient and health delays. The authors focused on ideas and therefore managed to set the stage for problem they were reporting. Although most of the literature is presented under the discussion of the results, a separate heading on literature review could have been added.

\section{Relevance}

The article identifies a wide gap in knowledge about cause, mode of transmission and duration of treatment of among TB and MDR-TB patients. It is written to inform researchers and scholars and not entertain. This is an academic journal on a credible academic data base. Majority of participants in this study first consulted private sector for treatment of TB and Cowan (2013) reports that this phenomenon is associated with switch over from one regiment to another. Enumerating patient and health provider delays among MDR-TB patients is crucial in a number of ways. The data provides information on possibility of higher deaths and dynamics in transmission and disease management at individual and at a community level (Yimer, Bjune \& Alene, 2005). Bjune (2005) asserts that assessing health seeking behavior among drug resistant TB patients is crucial in modeling the reproductive rate of the MDR-TB epidemic. MDR-TB is estimated to account for approximately $20 \%$ of the global TB mortality, with mortality rates approximated at $40 \%$ for MDR-TB patients and $60 \%$ among XDR-TB patient (WHO, 2015). Drug resistance TB also has a higher morbidity than drug sensitive TB.

Additionally, MDR-TB causes a strain on health system delivery because the disease is chronic in nature and there is a higher risk of transmission to health care workers (O'Donnell et al., 2010). There is also a serious challenge of the economic effect poised by MDR-TB. High costs of managing MDRTB are not sustainable in high burden countries as these countries are also among the poorest. This disrupts TB control. STOP TB Partnership has reported an anticipated shortfall in World resources (WHO, 2015). Marks and colleagues (2016) argue that in USA, the average inpatient costs for MDRTB patients is estimated to be around US81 000 and the figure is even outrageously high for XDR-TB patients which stands at $\$ 285000$ per year. TB is preventable if those infected with the bacillus promptly seek health care. However, even in most efficient and effective health systems, TB diagnosis and treatment delays are unavoidable.

\section{Objectivity}

The purpose of the study was to explore the knowledge about causes, mode of spread, duration of treatment among TB and MDR-TB patients. This objective was met, greatly supported with current literature and reliable sources. Tables and figures are clear and succinct and can be read easily for major findings by themselves there by achieving some objectivity. Limitations of a case control study are also stated. One weakness in the method used is in data analysis. Data could have been weighted prior to analysis to account for unequal probabilities of selection. Patient and health care system 
delays could also have been presented as the median (inter-quartile range) number of days. Analysis of data was supposed to detect significant differences in distributions of descriptive quantitative variables between cases and controls.

\section{Stability}

The article is published in IOSR Journal of Dental and Medical Sciences. IOSR Journal of Dental and Medical Sciences is one of the specialists Journals in Dental Science and Medical Science published by International Organization of Scientific Research (IOSR). The journal publishes papers of the highest scientific merit and widest possible scope of work in all areas related to medical and dental science.

\section{Analysis of graphs and tables}

Three figures and one table are used to summarize the data and the authors' figures are consistent with the format of currently accepted norms regarding data presentation. These figures and a table are necessary in order to tell the story of this research inquiry without taking much of space.

\section{Advance knowledge on the topic}

Drug sensitivity testing is the gold standard for testing resistance to TB drugs. However, the major drawback of culture-based methods to identify TB is that they are cumbersome and results are obtained after a long time. Detection of TB using culture-based methods can take 6 weeks or longer because the mycobacterium is a slow grower. Recently, there has been an improvement in molecular diagnostics, and this advancement has brought with it newer test methods for rapid TB detection and drugs sensitivity (Parsons et al, 2011). In the last 10 years, molecular techniques for the identification of TB and resistance genes are now available commercially. Most molecular technologies employ nucleic acid amplification tests (NAAT), based on the amplification of a short DNA or RNA strands of the bacillus. NAAT when employed to diagnostic specimen can reduce the turnaround time for laboratory diagnosis of TB by at least 2-4 weeks compared to traditional methods (Parsons et al, 2011). Molecular detection methods for TB resistance include line probe assays (LPA) and real-time PCR using GeneXpert Ultra.

UNITAID (2014) avers that in medium term, the need for a biomarker-based, low cost, non-sputum based test remains a key priority for diagnostic beyond the microscopy at peripheral level where most patients first seek help. New breakthrough in molecular diagnosis of TB using whole-genomic sequencing (WGS) has resulted in shorter time for detection and therefore faster and accurate treatments. A crucial method that allows WGS prior specimen culture has recently been discovered. WGS data can be readily obtained several weeks before drug sensitive test data is made available and this can be used to confirm rifampicin resistance from Xpert MTB/RIF assay. Gene sequencing also offers hope in understanding drug resistant TB and the mostly targeted sequence in TB organism is the insertion sequence IS6110 (Palomino, 2012).

In their study retrospective cohort study, Schnippel et al., (2018) reveal that new bedaquiline-based treatment regimens are attributable with significant decrease in deaths among patients with drugresistant TB, compared with the standard treatment. Based on these findings, as from July 2018, patients with MDR-TB in South Africa now receive life changing treatment with Bedaquiline. The roll-out of Bedaquiline is crucial as it replaces old treatment options which consist of injections administered drug resistant treatment. As such, South Africa has earned a top spot in TB drug resistant treatment in being the first country to roll out an injection-free regimen. Furthermore, Bedaquiline is a friendlier short regiment, which has the capacity to improve adherence and ensure success. Other countries with high burden of TB, HIV tuberculosis and drug resistant TB can take a leaf from South Africa's quantum leap.

\section{Conclusion}

The article by Dr Shiv Kuumar Yadav et al., (2015) is relevant as study findings indicate that there is requisite to improve the TB and MDR-TB knowledge on symptoms and modes of transmission in communities with high burden of the disease. This calls for policy development and implementation 
in areas like Information Education and Communication (IEC) and need to adopt the Behavior Change Communication (BCC) model at the community level by health care providers. Use of social marketing strategies tailor made for different population groups is recommended. The content, structure, strengths sand limitations of the article were evaluated and critiqued. The article adds to existing literature involving TB and MDR-TB health seeking behavior.

\section{References}

[1]. Bjune G. (2005). Tuberculosis in the $21^{\text {st }}$ century: an emerging pandemic. Nor Epidemiol; 15 (2):133-139.

[2]. Cowan J., Cowan J., Barnhart S., Demamu S., Fiseha D., Graham W. \& et al. (2013). A qualitative assessment of challenges to tuberculosis management and prevention in Northern Ethiopia. Int J Tuberc Lung Dis; 17:1071-5.

[3]. Field S.K., Fisher D., Jarand J.M., Cowie R.L. (2012). New treatment options for multidrug- resistant tuberculosis. The Adv Respir Dis;6: 255-268.

[4]. Finnie R.K.C., Khoza L.B., Van Den Borne B., Mabunda T., Abotchie P., Mullen P.D. (2011).

[5]. Factors associated with patient and health care system delay in diagnosis and treatment for TB in subSaharan African countries with high burdens of TB and HIV. Trop Med Int Health. 2011; 16:394-411.

[6]. Marks S.M., Hirsch-Moverman Y., Salcedo K. and the TB Epidemiologic Studies Consortium (2016). Characteristics and costs of multidrug-resistant tuberculosis in-patient care in the United States, 2005-2007. Int J Tuberc Lung Dis; 20:435-41.

[7]. Mavhu W., Dauya E., Banason T., Munyati S., Cowan F.M., Hart G., et al. (2010). Chronic cough and its association with TB-HIV co-infection: factors affecting help-seeking behavior in Harare, Zimbabwe. Trop Med Int Health. 15:574-9.

[8]. Nkosi D., Janssen S., Padanilam X., Louw R., Menezes C.N., Grobusch M.P. (2013). Factors influencing specialist care referral of multidrug and extensively drug-resistant tuberculosis patients in Gauteng/South Africa: a descriptive questionnaire based study. BMC Health Serv Res. Accessed on 1 September 2017; 13:268. doi:10.1186/1472-6963-13-268 PMID: 23837531.

[9]. O’Donnell M.R., Jarand J., Loveday M., et al. (2010). High incidence of hospital admissions with multidrug resistant and extensively drug-resistant tuberculosis among South African health care workers. Ann Intern Med; 153: 516-22.

[10]. Palomino J.C., Martin A., Von Groll A., Portaels F. (2008). Rapid culture-based methods for drug resistance detection in Mycobacterium tuberculosis. J Microbiol Methods;75: 161-166.

[11]. Parsons L.M., Somoskovi A., Gutierrez C., Lee E., Paramasivan C.N., et al. (2011). Laboratory diagnosis of tuberculosis in resource-poor countries: challenges and opportunities ClinMicrobiol Rev;24: 314-350.

[12]. Skordis-Worrall J., Hanson K., Mills A. (2010). Confusion, caring and tuberculosis diagnostic delay in Cape Town, South Africa; 14:171-80.

[13]. Schnippel, K et al., (2018). Effect of bedaquiline on mortality in South African patients with drug-resistant tuberculosis: a retrospective cohort study. Lancet Respir Med 2018 http://dx.doi.org/10.1016/S22132600(18)30235-2.

[14]. W.H.O. (2011). Global Tuberculosis control: WHO Report. Geneva: World Health Organization.

[15]. W.H.O. (2013). Global Tuberculosis Report. Geneva: World Health Organization.

[16]. W.H.O. (2015). Global Tuberculosis Report. Geneva: World Health Organization.

[17]. Yimer S., Bjune G., Alene G. (2005). Diagnostic and treatment delay among pulmonary tuberculosis patients in Ethopia: a cross sectional study. BMC Infect Dis. 5:112. Doi: 10.1186/1471-2334-5-112. Available from: http://www.biomedcentral.com/1471-2334/5/112. Download 02 October 2017. 